\title{
AUTO-TUNING OF FUZZY PID CONTROLLERS
}

\author{
P. J. Escamilla-Ambrosio and N. Mort \\ Department of Automatic Control and Systems Engineering \\ University of Sheffield \\ Mappin Street, Sheffield S1 3JD, United Kingdom \\ Phone: +44 (0) 114222 5619, Fax: +44 (0) 1142225661 \\ E-mail: COP99PJE@Sheffield.ac.uk
}

\begin{abstract}
In this work the auto-tuning procedure proposed by Astrom and Hagglund is extended and developed for tuning the scaling factors of a modified hybrid PID type fuzzy logic controller (MHPID-FLC). This new procedure is based on two steps. First, mathematical expressions to link the scaling factors of the MHPID-FLC with the proportional, integral and derivative actions of its traditional counterpart are derived. Second, based on this relationship and using the Ziegler-Nichols tuning formulae, the scaling factors of the MHPID-FLC are obtained by means of a relay experiment. The effectiveness of this approach is shown in benchmark processes taken from the literature. Copyright $(2002$ IFAC
\end{abstract}

Keywords: Autotuners, fuzzy PID control, PID control.

\section{INTRODUCTION}

In recent years fuzzy logic controllers (FLC) have been widely used for industrial processes exploiting their heuristic nature associated with simplicity and effectiveness for both linear and non linear systems. In particular, several structures of PID type FLC have been used (including PI and PD). As a consequence, research into this type of FLC has increased considerably. Lately, the research effort has been focused on the construction of an explicit link between the scaling factors of PID type FLC (PID-FLC) and the three actions of traditional PID control (TPID). The direct result of this link would bring the possibility of applying the systematic design and tuning methods of TPID control to design and tune PID-FLC.

Several approaches have been reported in the fuzzy control literature establishing a link between TPID and PID-FLC (Mann, et al., 2001; Li and Tso, 2000; $\mathrm{Xu}$, et al., 2000). However, these have often resulted in complicated mathematical expressions and, moreover, some of the parameters involved are heuristically established and this heuristic is not specified. Indeed, the task of constructing the link is not an easy one. First of all, several structures of PID-FLC have been proposed, and it is necessary to select the one most suitable for the construction of the link. Second, based on the chosen structure, a clear and explicit relationship between the parameters that define this structure with the three control actions of TPID control have to be derived. And finally, the systematic design and tuning methods of TPID control have to be translated for designing and tuning the selected PID-FLC structure.

Based on the investigation of the relationship between the gains of TPID control and the scaling factors of a modified hybrid PID-FLC (MHPIDFLC), in this paper a new methodology for designing and tuning PID-FLC is presented. First, in section 2, a review of the different structures of PID-FLC is carried out. Next, in section 3, the relationship between the proportional, integral and derivative actions from TPID control and the scaling factors of the MHPID-FLC is found through mathematical analysis and comparison. Then, in section 4, the auto- 
tuning methodology proposed by Astrom and Hagglund (1995, 1984) is extended and developed for automatically tuning the scaling factors of the MHPID-FLC. It is shown how the scaling factors can be directly derived from the Ziegler-Nichols frequency response method. As a result the performance of the MHPID-FLC will be better than, or at least as good as, that of its traditional counterpart. Next, in section 5, the viability of this approach is demonstrated by simulating several benchmark processes taken from the literature. Finally, in section 6, conclusions and perspectives of this work are given.

\section{PID-FLC STRUCTURES}

As in traditional control, in fuzzy control there are the analogous structures of the PI type fuzzy logic controller (PI-FLC), PD type fuzzy logic controller (PD-FLC) and the PID type fuzzy logic controller (PID-FLC). The PI-FLC and PD-FLC have been extensively studied (Lee, 1990; Lee, 1993; Jantzen, 1997; Tang and Mulholland, 1987), and have achieved wide acceptance in both academic research and industrial applications. However, the PID-FLC is considered to be still at its early stage of development (Driankov, et al., 1993; Li and Tso, 2000). This is shown by the numerous recent research papers reporting the exploration of different PID-FLC structures (Jantzen, 1999; Li and Tso 2000; Mann, et al., 1999; Woo, et al., 2000, Xu, et al., 2000).

Initially, the PID-FLC structures were designed considering three terms as inputs (Driankov, et al., 1993; Abdelnour, et al., 1991). Obviously, the rule base of these fuzzy controllers is three-dimensional (3-D), which makes it difficult to obtain since 3-D information is usually beyond the sensing capability of a human expert. To overcome this problem, the intuitive solution is the combination of a PI-FLC and a PD-FLC to form a PID-FLC. This idea has been developed basically in two ways, a parallel combination (PPID-FLC) and a hybrid combination (HPID-FLC).

The PPID-FLC structure was first proposed by $\mathrm{Li}$ and Gatland (1996), and lately has been studied by $\mathrm{Xu}$, et al., (2000). In this structure the three-term PID-FLC is divided into two separate PI and PD parts. Thus two rule bases are used, one for a PI-FLC and one for a PD-FLC; the output is obtained adding the respective crisp control output, as shown in figure 1. This structure has the advantage that both rule bases are two-dimensional avoiding the difficulty of designing a 3-D rule base. Consequently the design of a PID rule base becomes the design of both a PI and a PD rule bases. These two rule bases share the same inputs, which reduces the tuning complexity.

The HPID-FLC structure was first proposed by $\mathrm{Li}$ (1997), and lately has been studied by Mann, et al. (1999), Li and Tso (2000). In the HPID-FLC structure a common two-dimensional rule base is employed. This rule base is shared for both the PI-
FLC and the PD-FLC parts, as shown in figure 2. It means that, once appropriate scaling factors are selected, a PID control strategy is implemented by combining a PI incremental algorithm and a PD positional algorithm using a two-term fuzzy control rule base without any increase in the number of rules. This simplifies the PID-FLC structure as it is simpler, easier to implement and faster in computation. The PI rule base is selected as the one used, because PI control is normally more important for steady state behaviour.

In both PPID-FLC and HPID-FLC structures (see figures 1 and 2) GE and GCE are called the input scaling factors, while GU and GCU are called the output scaling factors; $T s$ is the sampling period of time. Inside these structures a fuzzy control system (FCS) develops the three well known processes of fuzzification, rule evaluation and defuzzification (Lee, 1990; Driankov, et al., 1993). The variables $e$ and $c e$ are the error and the change in error defined by:

$$
\begin{aligned}
e_{k} & =y_{r_{k}}-y_{k} \\
c e_{k} & =e_{k}-e_{k-1}
\end{aligned}
$$

where $y_{r}$ is the desired response and $y$ is the actual process response; $k$ is the sampling instance.

In this paper the HPID-FLC structure is selected as the one used for the purposes of this work. However, it is modified and several considerations are made, given in next section, in order to make comparisons with TPID control.

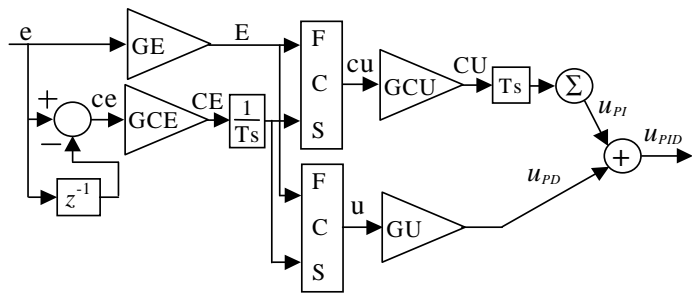

Fig. 1. PI-FLC + PD-FLC structure for a PPID-FLC.

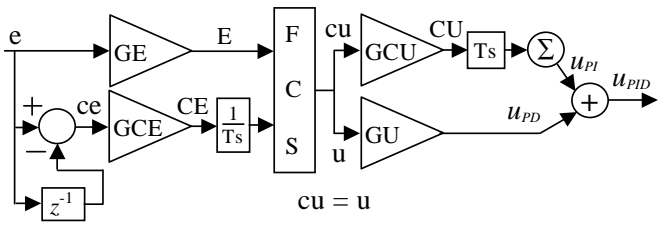

Fig. 2. Structure for an HPID-FLC.

\section{MATHEMATICAL ANALYSIS AND COMPARISON}

First, a TPID controller in digital form can be expressed as (to avoid confusion, in this work the symbol * means multiplication):

$$
\begin{aligned}
u_{P I D_{k}} & =K_{P} * e_{k}+K_{I} * \sum_{i=0}^{k} e_{i}^{*} T s+K_{D} * \frac{c e_{k}}{T s} \\
& =K_{P} *\left(e_{k}+\frac{1}{T_{i}} * \sum_{i=0}^{k} e_{i} * T s+T d * \frac{c e_{k}}{T s}\right)
\end{aligned}
$$


However, in order to avoid derivative kick in the implementation of (3) a modified derivative term is used. Additionally, when the Ziegler-Nichols tuning formula is applied a set-point weighting factor is employed to reduce overshoot (Astrom and Hagglund, 1995), thus (3) is transformed as follows,

$$
\begin{array}{r}
u_{P I D_{k}}=K_{P} *\left[\beta_{k} *\left(y_{r_{k}}-y_{k}\right)+\frac{1}{T_{i}} * \sum_{i=0}^{k} e_{i} * T s-T d * \frac{c y_{k}}{T s}\right] \\
=K_{P} * \beta_{k} * y_{r_{k}}-K_{P} y_{k}+K_{I} * \sum_{i=0}^{k} e_{i} * T s-K_{D} * \frac{c y_{k}}{T s}
\end{array}
$$

Observe in (4) that the variable ce in (3) has been replaced by $-c y_{k}$, where $c y_{k}$ is defined as,

$$
c y_{k}=y_{k}-y_{k-1}
$$

The incorporation of this last modification in the HPID-FLC structure modifies it as shown in figure 3 . This modified HPID-FLC (MHPID-FLC) structure is the one used here.

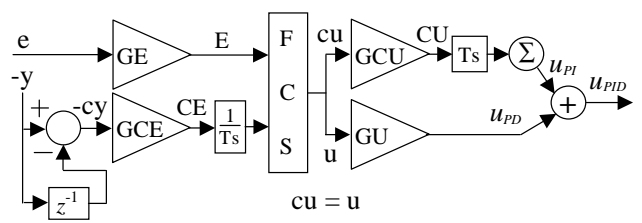

Fig. 3. Modified HPID-FLC structure.

Next, if the following assumptions are made:

1. The FCS inside the MHPID-FLC structure is a first-order Sugeno fuzzy model (Takagi and Sugeno, 1985), with fuzzy rules of the form:

$$
\text { If } E \text { is } \mathrm{A} \text { and } C E \text { is } \mathrm{B} \text { then } \mathrm{u}=p^{*} E+q^{*} C E+r
$$

where A and B are fuzzy sets in the antecedent, while $p, q$, and $r$ are all constants.

2. The FCS rule base consists of four rules,

$R_{l}$ : If $E$ is $\mathrm{N}$ and $C E$ is $\mathrm{N}$ then $\mathrm{u}=p_{1} * E+q_{1} * C E+r_{l}$ $R_{2}$ : If $E$ is $\mathrm{N}$ and $C E$ is $\mathrm{P}$ then $\mathrm{u}=p_{2}{ }^{*} E+q_{2}{ }^{*} C E+r_{2}$ $R_{3}$ : If $E$ is $\mathrm{P}$ and $C E$ is $\mathrm{N}$ then $\mathrm{u}=p_{3} * E+q_{3} * C E+r_{3}$ $R_{4}$ : If $E$ is $\mathrm{P}$ and $C E$ is $\mathrm{P}$ then $\mathrm{u}=p_{4}{ }^{*} E+q_{4}{ }^{*} C E+r_{4}$

where the coefficient constants $p_{i}=q_{i}=1$, and $r_{i}=0$; for $i=1,2,3,4$.

3. The universe of discourse for both FCS inputs is normalised on the range $[-1,1]$.

4. The membership functions of the input variables, $E$ and $C E$, to the FCS are triangular complementary adjacent fuzzy sets (Escamilla, 1999; Gravel and Mackenberg, 1995), and they are defined as shown in figure 4(a). The fuzzy labels means, $\mathrm{P}=$ Positive, and $\mathrm{N}=$ Negative.

5. The product-sum compositional rule of inference (Kosko, 1992) is used in the stage of rule evaluation. 6. The weighted average is used in the defuzzification process.

then the FCS inside the MHPID-FLC structure is the simplest that can be considered, and its output is given by the sum of its inputs. This FCS is known as the normalised and linear FCS (Jantzen, 1999); its control surface is shown in figure 4(b).

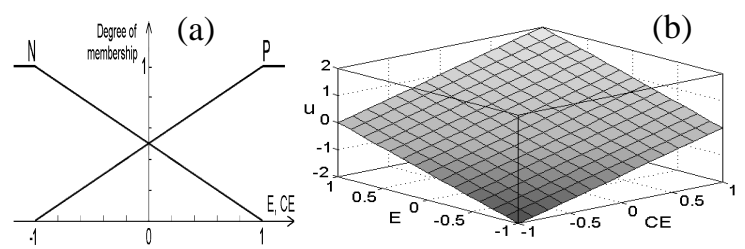

Fig. 4. (a) Membership functions for E and CE; (b)

Control surface of the normalised and linear FCS.

Thus, under the assumptions 1 to 6 , the PI-FLC and the PD-FLC output parts (see figure 3) of the MHPID-FLC are given as,

$$
\begin{aligned}
u_{P I_{k}} & =\sum_{i=0}^{k}\left(c u_{i} * G C U * T s\right)=G C U * T S * \sum_{i=0}^{k} c u_{i} \\
& =G C U * T s * \sum_{i=0}^{k}\left[G E * e_{i}-G C E * \frac{c y_{i}}{T s}\right] \\
u_{P D_{k}} & =u_{k} * G U=G U *\left[G E * e_{k}-G C E * \frac{c y_{k}}{T s}\right]
\end{aligned}
$$

Then the MHPID-FLC output $u_{P I D}$ is given by adding (6) and (7),

$$
\begin{aligned}
u_{P I D_{k}}= & u_{P I_{k}}+u_{P D_{k}} \\
= & G C U * G E * \sum_{i=0}^{k} e_{i} * T s-G C U * G C E * \sum_{i=0}^{k} c y_{i} \\
& +G U * G E * e_{k}-G U * G C E * \frac{c y_{k}}{T s} \\
= & G C U * G E * \sum_{i=0}^{k} e_{i} * T s-G C U * G C E * y_{k} \\
+ & G U * G E *\left(y_{r_{k}}-y_{k}\right)-G U * G C E * \frac{c y_{k}}{T s} \\
= & G U * G E * y_{r_{k}}-(G C U * G C E+G U * G E) * y_{k} \\
+ & G C U * G E * \sum_{i=0}^{k} e_{i} * T s-G U * G C E * \frac{c y_{k}}{T s}
\end{aligned}
$$

Comparing (4) and (8) it is noted that the MHPIDFLC works like a TPID controller with set-point weighting factor and modified derivative term. The equivalent set-point weight, proportional, integral, and derivative components are:

$$
\begin{aligned}
& K_{P} * \beta=G U * G E \\
& K_{P}=G C U * G C E+G U * G E \\
& K_{I}=\frac{K_{P}}{T_{i}}=G C U * G E \\
& K_{D}=K_{P} * T_{d}=G U * G C E
\end{aligned}
$$

This means that the scaling factors of the MHPIDFLC can be derived from the proportional, integral and derivative gains obtained for the traditional PID controller using well known methods, i. e. the Ziegler-Nichols tuning method (Astrom and Hagglund, 1995). A procedure for this task is presented in next section. 


\section{AUTO-TUNING OF THE SCALING FACTORS OF THE MHPID-FLC}

If the values of $K_{P}, K_{I}$, and $K_{D}$ are available, then the values of GE, GCE, GU and GCU in the MHPIDFLC structure can be calculated in the following way. The proportional gain given by (10) can be separated in two parts:

$$
\begin{aligned}
K_{P} & =G C U * G C E+G U * G E \\
& =\alpha * K_{P}+(1-\alpha) * K_{P}
\end{aligned}
$$

from here it follows,

$$
\begin{aligned}
& G C U * G C E=\alpha * K_{P} \\
& G U * G E=(1-\alpha) * K_{P}
\end{aligned}
$$

From (9) and (15) it can be directly deduced that,

$$
\beta=1-\alpha
$$

From assumption 3 it is clear that the possible values of $E$ are in the range $[-1,1]$, thus in order to avoid saturation, GE is selected as:

$$
G E=1
$$

In consequence, from (17), (15) becomes,

$$
G U=(1-\alpha) K_{P}
$$

In a similar way, from (17), (11) becomes,

$$
G C U=K_{I}
$$

Calculating GCE from (12) gives,

$$
G C E=\frac{K_{D}}{G U}
$$

and from (18) in (20a) gives,

$$
G C E=\frac{K_{D}}{G U}=\frac{K_{D}}{(1-\alpha) * K_{P}}
$$

Thus, once the parameter $\alpha$ is defined, the scaling factors can be calculated using Equations (18) to (20). But now the question is how should the parameter $\alpha$ be properly defined? First of all $\alpha$ has to satisfy (14) and (18), thus from (19) and (20b) in (14) gives,

$$
K_{I} * \frac{K_{D}}{(1-\alpha) * K_{P}}=\alpha * K_{P}
$$

and solving (21) for $\alpha$ gives,

$$
-\alpha^{2}+\alpha=\frac{K_{I} * K_{D}}{K_{P}^{2}}
$$

But, from TPID control,

$$
K_{I}=\frac{K_{P}}{T_{i}} \quad ; \quad K_{D}=K_{P} * T_{d}
$$

Thus, from (23) in (22) gives,

$$
-\alpha^{2}+\alpha-\frac{T_{d}}{T_{i}}=0
$$

and applying the relation between $T_{i}$ and $T_{d}$ given by the Ziegler-Nichols frequency response tuning method (see Table 1), finally leads to,

$$
-\alpha^{2}+\alpha-\frac{1}{4}=0
$$

Solving equation (25) results in,

$$
\alpha_{1}=\alpha_{2}=\frac{1}{2}
$$

Finally, by substituting the value of $\alpha$ in (18) and (20b), the solutions for GU and GCE become straightforward.

The previous development means that the MHPIDFLC is equivalent to its traditional counterpart given by (4) when $\beta$ is selected as 0.5 , calculated from (16), and the Ziegler-Nichols frequency response method is used to tune the controller. Additionally, this means that based on this development, the relay autotuning algorithm for TPID control (Astrom and Hagglund, 1984; 1995) can be extended and developed to tune the scaling factors of the MHPIDFLC as is explained next. First of all the scaling factors have to be a function of $K_{u}$ and $T_{u}$, thus from (18), (19), and (20) gives,

$$
\begin{aligned}
& G U=\frac{K_{P}}{2}=\frac{0.6 * K_{u}}{2}=0.3 * K_{u} \\
& G C U=\frac{K_{P}}{T_{i}}=\frac{0.6 * K_{u}}{0.5 * T_{u}}=1.2 * \frac{K_{u}}{T_{u}} \\
& G C E=2 * \frac{K_{P} * T_{d}}{K_{P}}=2 * T_{d}=\frac{2}{8} * T_{u}=\frac{1}{4} * T_{u}
\end{aligned}
$$

Table 1 PID parameters according to the ZieglerNichols frequency response method

\begin{tabular}{ccc}
$K_{P}$ & $T_{i}$ & $T_{d}$ \\
\hline $0.6 * \mathrm{~K}_{\mathrm{u}}$ & $(1 / 2)^{*} T_{u}$ & $(1 / 8)^{*} T_{u}$ \\
\hline
\end{tabular}

A summary of the relationships between the scaling factors of the MHPID-FLC, the gains of its traditional counterpart and the Ziegler-Nichols tuning frequency response method are given in Table 2 . The value of the parameters $K_{u}$ and $T_{u}$, called the "ultimate gain" and "ultimate period" respectively, can be obtained from a relay feedback experiment as shown in figure 5 . Therefore, the ultimate gain and the ultimate frequency can be calculated from this experiment as,

$$
K_{u}=\frac{4 * h}{\pi * a} ; \quad T_{u}=\frac{2 * \pi}{\omega_{u}}
$$

where $h$ is the relay amplitude, $a$ is the process output amplitude, and $\omega_{u}$ is the oscillation frequency of the process output. It has been shown by Astrom and Hagglund (1984) that the simple estimation of $K_{u}$ and 
$T_{u}$ based on zero-crossing and peak detection works very well. Thus this method is used in this work and the values found are used to calculate the scaling factors of the MHPID-FLC.

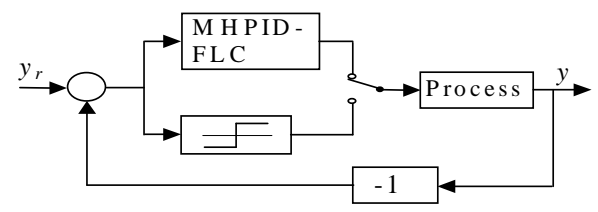

Fig. 5 Relay feedback experiment.

Table 2 Relationship between the scaling factors of the MHPID-FLC, the TPID control gains, and the Ziegler Nichols frequency response tuning formulae

\begin{tabular}{ccccc} 
B a sed on & G E & G C E & G U & G C U \\
\hline$K_{u}, T_{u}$ & 1 & $\frac{1}{4} * T_{u}$ & $0.3 * K_{u}$ & $1.2 * \frac{K_{u}}{T_{u}}$ \\
$K_{P}, K_{I}$, & & $2 * \frac{K_{D}}{K_{P}}$ & $\frac{1}{2} * K_{P}$ & $K_{I}$ \\
$K_{D}$ & 1 & & $\frac{1}{2} * K_{P}$ & $\frac{K_{P}}{T_{i}}$ \\
$K_{P}, T_{i}$, & 1 & $2 * T_{d}$ & \\
$T_{d}$ & 1 & &
\end{tabular}

\section{SIMULATION AND COMPARISONS}

In this section the viability of this approach is demonstrated by simulating several benchmark processes taken from the literature. Three auto-tuning experiments for each process have been developed in Matlab environment, together with Simulink and the Fuzzy Logic Toolbox. The first experiment is developed to simulate a relay auto-tuning procedure for the MHPID-FLC. Here the scaling factors are obtained applying the formulae given in Table 2 . The second and the third experiments use the same procedure but now to tune the gains of the TPID given by equation (3), and the TPID given by equation (4) (referred to as TPID2) with a set-point weighting factor of 0.5 . In these cases the tuning formulae given in Table 1 is applied.

After a relay experiment a unit step and a unit load perturbation are introduced on the processes in order to observe their responses. The process responses under auto-tuned MHPID-FLC, TPID and TPID2 control are plotted and compared for each case as is described next:

1) First-order plus dead time process (Hang, et al., 1991):

$$
G_{1}(s)=\frac{e^{-0.2 s}}{(s+1)}
$$

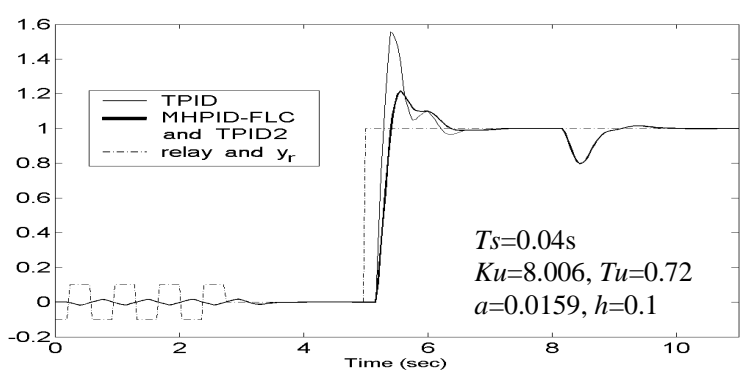

Fig. 6. Comparison of set-point and load disturbance responses for $\mathrm{G}_{1}(\mathrm{~s})$.
2) Second-order plus dead time process (Hang, et al., 1991; Zhuang and Atherton, 1993):

$$
G_{2}(s)=\frac{e^{-0.4 s}}{(s+1)^{2}}
$$

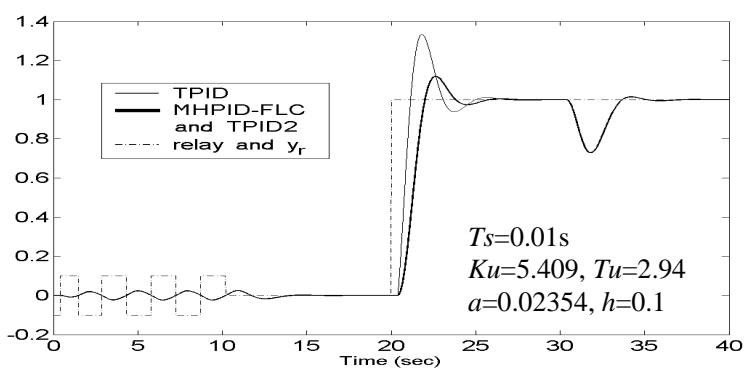

Fig. 7. Comparison of set-point and load disturbance responses for $\mathrm{G}_{2}(\mathrm{~s})$.

3) High-order process (Zhao, et al., 1993):

$$
G_{3}(s)=\frac{27}{(s+1)(s+3)^{3}}
$$

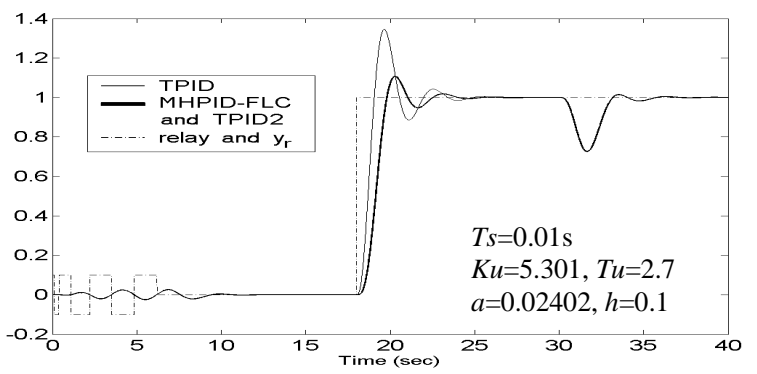

Fig. 8. Comparison of set-point and load disturbance responses for $\mathrm{G}_{3}(\mathrm{~s})$.

4) Non-minimum phase process (Hang, et al., 1991):

$$
G_{4}(s)=\frac{1-1.4 s}{(s+1)^{3}}
$$

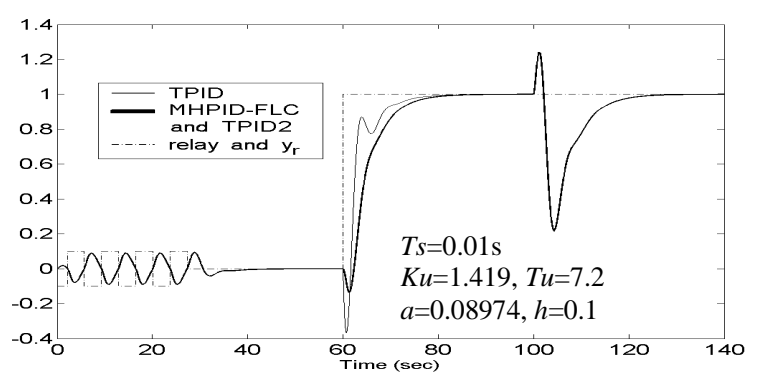

Fig. 9. Comparison of set-point and load disturbance responses for $\mathrm{G}_{4}(\mathrm{~s})$.

From the results observed in figures 6 to 9 it is noted that the overshoot in the step-response (undershoot for process $\mathrm{G}_{4}$ ) is excessive when TPID control is used. But, the overshoot is reduced by approximately $60 \%$ when MHPID-FLC and PID2 control are employed. However, this reduction in overshoot is accompanied by a small reduction in the speed of response (the rise time increases). Note that exactly the same response is obtained when MHPID-FLC and TPID2 control are used. Thus it is proved that the MHPID-FLC is equivalent to its traditional counterpart given by (4) when $\beta$ is selected as 0.5 . This means that the set-point weighting factor is 
embedded in the MHPID-FLC structure. Note that in all cases the load disturbance rejection is the same. Thus the MHPID-FLC and the TPID2 controllers sacrifice speed of response to a far smaller degree in order to obtain a substantial reduction in overshoot with respect to TPID control. However, this does not affect the load disturbance response.

\section{CONCLUSIONS}

A new methodology for designing and auto-tuning the scaling factors of a modified hybrid PID type fuzzy logic controller (MHPID-FLC) has been presented. This procedure has been derived from the establishment of a relationship between the three actions of traditional PID control and the scaling factors of the MHPID-FLC. A set of formulae were derived to calculate the scaling factors of the MHPID-FLC employing the Ziegler-Nichols frequency response method. It was proved that the MHPID-FLC works like a TPID controller with setpoint weighting factor of 0.5 and modified derivative term. A remarkable point is that, based on the established relationship, the systematic design and tuning methods of TPID control can be extended and developed for applications for designing and tuning of the MHPID-FLC.

The proposed methodology was tested in several simulated benchmark processes. In all cases the MHPID-FLC performance is equivalent to its traditional counterpart with a set-point weighting factor of 0.5 and modified derivative term. Thus, the set-point weighting factor is embedded in the MHPID-FLC structure; it is not necessary to specify it as another variable. However, in this case it is a fixed value $(0.5)$.

In this study the improvement of the MHPID-FLC by changing the values of the scaling factors or introducing nonlinearities in the FCS was not explored. This opens an avenue of investigation that is being explored by the authors.

\section{REFERENCES}

Abdelnour, G.M., C.H. Chang, F.H. Huang and J.Y. Cheung (1991). Design of a fuzzy controller using input and output mapping factors. IEEE Trans. Syst., Man, Cybern., 21, 952-960.

Astrom, K.J. and T. Hagglund (1984). Automatic tuning of simple regulators with specifications on phase and amplitude margins. Automatica, 20, 645-651.

Astrom, K.J. and T. Hagglund (1995). PID Controllers: Theory, Design, and Tuning, $2^{\text {nd }}$ edition. Instrument Society of America, USA.

Driankov, D., H. Hellendoorn, and M. Reinfrank (1996). An Introduction to Fuzzy Control. Springer-Verlag, New York.

Escamilla, P.J. (1999). Exposition and Test of a New Method of Defuzzification for Fuzzy Control Systems. Proc. IASTED International Conference on Control and Applications, July 24-26, Banff, Alberta, Canada, 503-508.

Gravel, A. and H. Mackenberg (1995). Mathematical analysis of the Sugeno controller leading to general design rules. Fuzzy Sets and Systems, 85, 165-175.

Hang, C.C., K.J. Astrom and W. K. Ho (1991). Refinements of the Ziegler-Nichols tuning formula. IEE Proceedings-D, 138, 111-118.

Jantzen, J. (1997). A robustness study of fuzzy control rules. In: EUFIT (ed.), Proc. Fifth European Congress on Fuzzy and Intelligent Technologies, ELITE Foundation, Promenade 9, D-52076 Aachen, 1222-1227.

Jantzen, J. (1999). Tuning of fuzzy PID controllers. Online 98-H-871 (fpid), Technical University of Denmark: Dept. of Automation, http://www.iau.dtu.dk/\#jj/pubs.

Kosko, B. (1992). Neural Networks and Fuzzy Systems: A dinamical Approach to Machine Intelligence. Prentice Hall, USA.

Lee, C.C. (1990). Fuzzy Logic in Control Systems: Fuzzy Logic Controllers, Parts I and II. IEEE Trans. Syst., Man, Cybern., 20, 404-435.

Lee, J. (1993). On methods for improving performance of PI-type fuzzy logic controllers. IEEE Trans. Fuzzy Syst., 1, 298-301.

Li, H.-X. and H.B. Gatland (1996). Conventional fuzzy control and its enhancement. IEEE Trans. Syst., Man, Cybern., Part B, 26, 791-797.

Li, H.-X. (1997). A comparative design and tuning for conventional fuzzy control. IEEE Trans. Syst., Man, Cybern., Part B, 27, 884-889.

Li, H.-X. and S.K. Tso (2000). Quantitative design and analysis of fuzzy proportional-integralderivative control-a step towards autotuning. Int. J. Syst. Sci., 31, 545-553.

Mann, G.K.I, B.G. Hu and R.G. Gosine (1999). Analysis of direct action fuzzy PID controller structures. IEEE Trans. Syst., Man, Cybern., Part $B, 29,371-388$.

Mann, G.K.I, B.G. Hu and R.G. Gosine (2001). Twolevel tuning of fuzzy PID controllers. IEEE Trans. Syst., Man, Cybern., Part B, 31, 263-269.

Takagi, T. and M. Sugeno (1985). Fuzzy identification of systems and its applications to modeling and control. IEEE Trans. Syst., Man, Cybern., SMC-15, 116-132.

Tang, K.L. and R.J. Mulholland (1987). Comparing fuzzy logic with classical controller designs. IEEE Trans. Syst., Man, Cybern., SMC-17, 10851087.

Woo, Z.-W., H.-Y. Chung and J.-J. Lin (2000). A PID type fuzzy controller with self-tuning scaling factors. Fuzzy Sets and Systems, 115, 321-326.

Xu, J.X., C.C. Hang and C. Liu (2000). Parallel structure and tuning of a fuzzy PID controller. Automatica, 36, 673-684.

Zhao, Z.-Y., M. Tomizuka and S. Isaka (1993). Fuzzy gain scheduling of PID Controllers. IEEE Trans. Syst., Man, Cybern., 23, 1392-1398.

Zhuang, M. and D.P. Atherton (1993). Autotuning of optimum PID controllers. IEE Proceedings-D, 140, 216-224. 